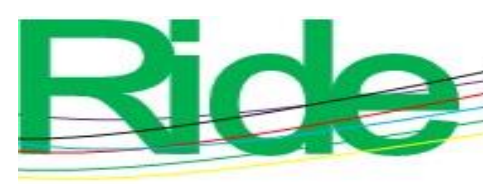

Revista lberoamericana para la

Investigación y el Desarrollo Educativo ISSN $2007-7467$

https://doi.org/10.23913/ride.v10i19.492

Artículos Científicos

\title{
El desarrollo profesional de docentes. Una propuesta desde la complejidad
}

The Professional Development of Teachers. A Proposal From the Complexity

O desenvolvimento profissional de professores. Uma proposta da complexidade

María Elena López Serrano

Escuela Normal de Atlacomulco "Profra. Evangelina Alcántara Díaz", México malyels@gmail.com https://orcid.org/0000-0003-0910-2428

\section{Resumen}

Pugnar por la mejora educativa es una constante en la política de todos los países. Y uno de los aspectos nodales son los docentes. La propuesta que aquí se presenta, desde una perspectiva teórica compleja, reconoce a estos como sujetos históricos que al incorporarse a las instituciones se construyen y reconstruyen. El planteamiento recupera tres ámbitos centrales: personal, profesional y social. El primero alude a la motivación, satisfacción y aprendizaje; el segundo reconoce la necesidad de propiciar desde las instituciones condiciones de comunicación y toma de acuerdos y espacios para la reflexión y las comunidades de aprendizaje. Finalmente, el social se asocia con el reconocimiento de la profesión y su relación con el desarrollo comunitario que redunde en pro de la sociedad. Los planteamientos de este artículo son producto de una investigación desarrollada de 2011 a la fecha. De manera específica presenta los resultados de dos proyectos: una investigación etnográfica denominada "El desarrollo profesional de los formadores de docentes. Una 


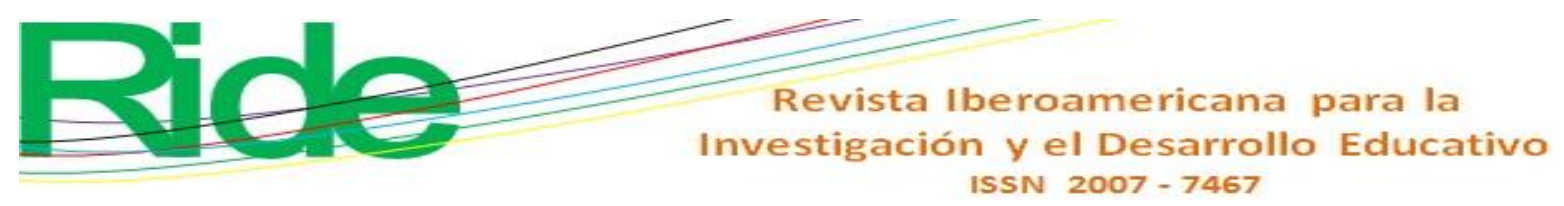

mirada desde la complejidad" y un proyecto de corte hermenéutico denominado "El desarrollo profesional docente al debate".

Palabras clave: complejidad, desarrollo profesional, enseñanza.

\begin{abstract}
Fighting for better education is a constant feature in every country's public policy, being focus on educators one of the main components in such policies. This proposal recognizes educators as historical beings, which, once incorporated to educational institutions, are constructed and reconstructed in personal, professional and social manners. These three central areas are discussed from a complex perspective throughout the proposal. The first aspect refers to motivation, satisfaction and learning. The second recognizes the need for improvement of conditions for communication, making agreements, spaces for reflection and learning communities. Finally, the third aspect describes the role of education for community development that benefits the society. This approach was based on two research projects' results: "Professional development of teacher educators: a complex view" and "Debating the teacher's professional development".
\end{abstract}

Keywords: complexity, professional development, teaching.

\title{
Resumo
}

Lutar pela melhoria educacional é uma constante na política de todos os países. E um dos aspectos nodais é o dos professores. A proposta apresentada aqui, a partir de uma perspectiva teórica complexa, reconhece-os como sujeitos históricos que, quando incorporados às instituições, são construídos e reconstruídos. A abordagem recupera três áreas centrais: pessoal, profissional e social. O primeiro refere-se à motivação, satisfação e aprendizado; o segundo reconhece a necessidade de promover, a partir das instituições, condições de comunicação e realização de acordos e espaços de reflexão e aprendizagem das comunidades. Por fim, o social está associado ao reconhecimento da profissão e sua relação com o desenvolvimento comunitário que resulta em benefício da sociedade. As abordagens deste 


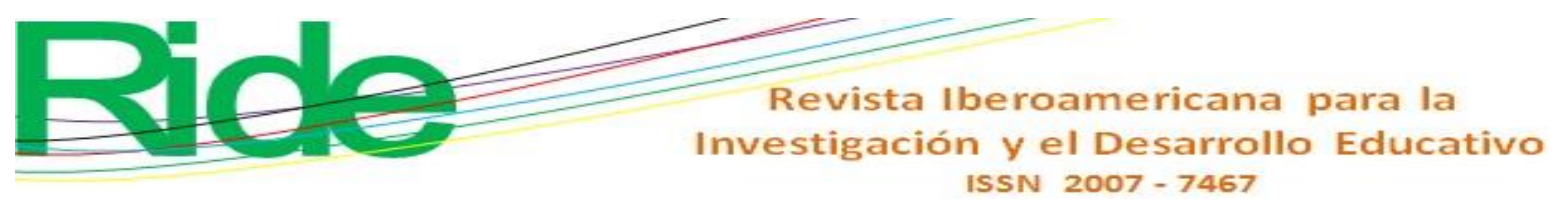

artigo são o produto de uma pesquisa desenvolvida de 2011 até hoje. Apresenta especificamente os resultados de dois projetos: uma pesquisa etnográfica denominada "O desenvolvimento profissional de formadores de professores". Um olhar da complexidade "e um projeto hermenêutico chamado" Desenvolvimento profissional do professor para debater".

Palavras-chave: complexidade, desenvolvimento profissional, ensino.

Fecha Recepción: Enero 2019

Fecha Aceptación: Junio 2019

\section{Introducción}

El desarrollo profesional en las diferentes esferas laborales implica el reconocimiento de la persona y de las posibilidades de su desempeño. De manera general, se reconocen dos formas de concebirlo: la primera se vincula directamente con la habilitación y capacitación para obtener mejores resultados que redunden en las instituciones. En esta visión, el ser humano se convierte en un recurso al que se le requiere invertir para el logro de los objetivos. Esto implica que las personas pueden ser moldeadas y modificar sus comportamientos en pro de los objetivos institucionales.

La otra postura reconoce a las personas como sujetos históricos que poseen conocimientos, habilidades, creencias e intereses que, al incorporarse y ser parte de una institución, contribuyen en su configuración, al tiempo que la organización también les da elementos para construirse o reconstruirse. Esta posición recupera planteamientos desde las teorías administrativas humanistas hasta las denominadas de sistemas alejados del equilibrio o sistemas complejos adaptativos de transacciones, que tienen que ver con la posibilidad de las organizaciones de autoorganizarse y lograr adaptarse. Lo que implica que hay que ajustarse al entorno y a sus características.

Como resultado de la investigación llevada a cabo, se plantea la siguiente propuesta para conceptuar el desarrollo profesional: "Proceso complejo que se construye de acciones colectivas e individuales, resultado del tejido de factores personales, sociales e institucionales, que contribuyen al logro de la realización profesional, posibilitando la

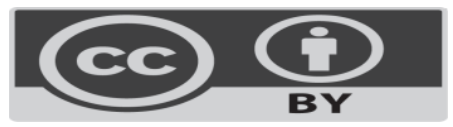




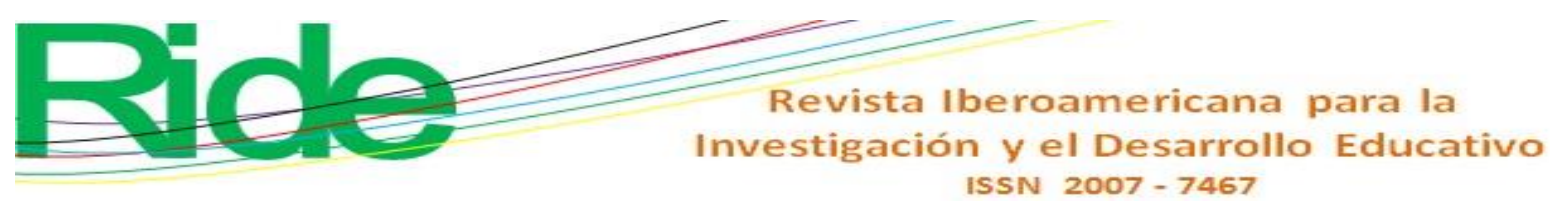

satisfacción de las necesidades de desarrollo personal, institucional y social” (López, 2013, p. 4).

A partir de una investigación desarrollada desde 2011 y que se ha integrado de manera preponderante por dos proyectos: "El desarrollo profesional de los formadores de docentes. Una mirada desde la complejidad" y "El desarrollo profesional docente al debate", cuyos resultados revelaron aspectos que dan pauta para materializar una propuesta con miras a que en las instituciones se consiga favorecer el desarrollo profesional de los docentes; a partir de dicho trabajo, como se decía, se estructura el planteamiento que se presenta en este documento. De ninguna manera es una guía estricta, sino más bien permite reconocer una serie de elementos que pueden ser considerados y a partir de los cuales se consiga potenciar el desarrollo profesional.

Así, pues, con la finalidad de analizar los aspectos considerados en el desarrollo profesional, se reconocen tres ámbitos: personal, institucional y social; no porque sean aislados sino debido a que permiten profundizar en sus relaciones y posibilidades.

\section{Método}

Si bien la propuesta que aquí se presenta es resultado de las dos investigaciones arriba mencionados, los principios generales que sirvieron de guía, indistintamente de los proyectos, parten de una postura que se afianza en el reconocimiento de que la realidad es una construcción realizada por los sujetos; motivo por el cual indagarla: recuperar su voz, la de los sujetos, da pauta para reconocerla. En este sentido, y en congruencia con el método, se consideró lo planteado por Walter Runciman (citado en Guber, 2011), quien divide el proceso de comprensión en tres niveles. En el primero, denominado primario o reporte, se da cuenta de lo ocurrido ("el qué"); el segundo, titulado la explicación o comprensión secundaria, alude a sus causas ("por qué"); y el tercero, la descripción o comprensión, se ocupa de lo que ocurrió desde la perspectiva de sus agentes (“el cómo es para ellos").

El primer proyecto desarrollado de 2011 a 2013 fue un estudio etnográfico que permitió delinear lo que se puede concebir como desarrollo profesional, a partir del reconocimiento de los sujetos históricos que intervienen en él, valorando que poseen

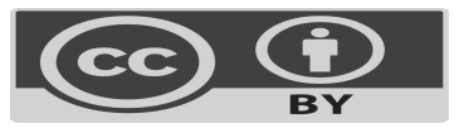




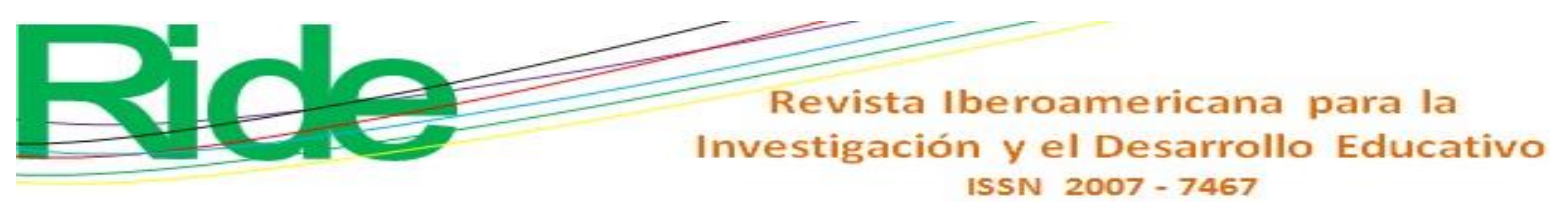

habilidades, experiencias y actitudes que al integrarse a las instituciones convergen y construyen nuevas perspectivas. Sin embargo, en esta investigación se reconoció la necesidad de profundizar en el análisis teórico y normativo. En consecuencia, se planteó un segundo trabajo en el que se recurrió a la hermenéutica y se ahondó en la revisión teórica y de otras investigaciones con miras a conformar un planteamiento que señalara un camino para que las instituciones, a partir del reconocimiento de su realidad, consiguieran hacer propuestas para que los profesionales de la educación fortalecieran o incrementaran su desarrollo profesional.

\section{Resultados}

En un primer momento resulta conveniente reconocer cómo concebir la complejidad, debido a que si bien ha sido planteada por autores como Immanuel Kant (1781), Darwin (1882), Hegel (1970), no es hasta finales del siglo XX cuando de manera más puntual se desarrolla una propuesta para responder a las necesidades sociales. En este documento se recuperan tres posturas. El pensamiento complejo y las teorías de sistemas y el caos. La primera y segunda reconocen que los sistemas son más que la mera unión de las partes debido a que la articulación de los elementos genera relaciones en las que surgen nuevas interacciones y algunas características se pierden, así como el reconocimiento de que el todo está en las partes y las partes en el todo (Ackoff, 1999; Morin, 1990). De la tercera, la propuesta del caos, se rescata que en los procesos complejos, a pesar de tener las mismas entradas, la salida es diferente debido a que se desconoce qué es lo que pasa en la "caja negra", motivo por el cual resulta poco factible pensar en soluciones únicas o en comportamientos uniformes y causales para este tipo de sistemas (Prigogine, 1999).

La complejidad da pauta para reconocer que el desarrollo profesional no es un sistema lineal sino uno caótico; uno en el que resulta necesario vislumbrar que no existen soluciones lineales y únicas para favorecerlo. Desde esta perspectiva, se efectúa la propuesta que se presenta y puntualizan elementos para plantear acciones que coadyuven a fortalecerlo, en el entendido de que serán procesos de construcción tanto colectivos como individuales. 

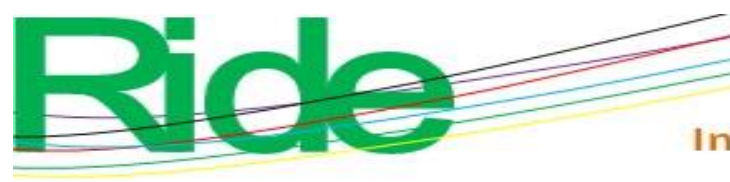

Revista Iberoamericana para la
Investigación y el Desarrollo Educativo
ISSN $2007-7467$

Para analizar los engranajes que se asocian con el desarrollo profesional, estos se agruparon en tres grandes ámbitos: personal, social y profesional. Con respecto al profesional, es necesario reflexionar lo que Day (2007), citando a Hargreaves, enuncia como una de las características de las escuelas creadoras:

Una cultura de compromiso y de entusiasmo por el perfeccionamiento continuo; que estimulen las relaciones informales en vez de las jerárquicas, y la diversidad en vez de la uniformidad entre el personal, y que muestren la disposición a fomentar todo lo que suponga experimentar con ideas nuevas, en una cultura en la que los errores se consideren vías para el aprendizaje. ( $\mathrm{p}$. 17).

Lo anterior reconoce que el trabajo en las instituciones se logra a partir de consolidar un aprendizaje continuo, lo cual es congruente con lo que se fomenta en los alumnos y da respuesta a las transformaciones sociales.

En el caso de la formación, no hay excepción, y, por el contrario, requiere que los docentes, de manera continua, estén actualizándose respecto a lo que sucede en el entorno, de las preocupaciones, riesgos y necesidades de las nuevas generaciones. Pero, ¿cómo conseguirlo en el marco de reformas curricular, exigencias administrativas y problemas personales? El diálogo entre pares y la reflexión de la práctica representan un medio para lograrlo. Aunque no es un proceso sencillo, iniciar con el reconocimiento de las necesidades y cómo a partir del análisis conjunto se logra llegar a consensos que enriquezcan el quehacer docente será un primer paso. Para ello se sugiere focalizar algunos aspectos asociados con el trabajo institucional que representan un punto clave a considerar.

El primer aspecto vinculado con ello es el desarrollo de una comunicación efectiva entre sus integrantes. Es valioso señalar que se vislumbran dos tipos de comunicación manifiestas en las instituciones. La primera tiene que ver con la toma de acuerdos para el desarrollo del trabajo cotidiano, las actividades inherentes a la organización, donde se concretan estándares de medición a los que se debe de dar respuesta. A este tipo de comunicación se le puede calificar como necesaria. 
La segunda es la comunicación institucional a través de la cual se toman acuerdos, se dialogan los logros, aciertos y desaciertos, se analizan las acciones, se promueve el beneficio común y el desarrollo de sus integrantes. En ella, la expresión libre de ideas permite construir planteamientos para ser puestos en práctica; se da voz a quienes realizan la tarea educativa y se propicia el análisis teórico y empírico, y así, en suma, se promueve el análisis y la reflexión del hacer cotidiano.

Otro aspecto tiene que ver los elementos institucionales. Se trata de generar comunidades donde el trabajo colaborativo sea una fortaleza, donde se desarrolle un trabajo con pleno convencimiento, todo lo cual requiere de tiempo y de espacios destinados para ello. En este sentido, resulta pertinente reconocer que si bien establecer metodologías o estrategias para ordenar los procesos desde lo administrativo permite establecer medios y mecanismos de seguimiento, en ocasiones se vuelven una camisa de fuerza en la que pareciera que hay aspectos que están delineados o estructurados y no es posible recuperar la experiencia de los actores educativos. Frente a este escenario, vale la pena que, antes de iniciar con los trabajos conjuntos, en un primer momento se busque consolidar los equipos de trabajo y de manera posterior se establezcan los procesos administrativos para regularlos.

Aunado a lo anterior, será necesario reconocer que las reuniones de los colectivos docentes tienen que promover el diálogo abierto sobre temas que sean comunes y, de esta manera, evitar caer en la monotonía. Para ello se plantea que las agendas sean definidas de forma común, que sean modificadas de acuerdo con el avance que se tenga o los nuevos retos que se presenten, pero, sobre todo, que el análisis no sea desde la perspectiva de quien ostenta el poder, sino que el diálogo y la confrontación de ideas permitan a los integrantes del colectivo sentirse libres de expresar sus puntos de vista, que al tiempo que recuperan el conocimiento empírico lo enriquezcan con fundamentos que den soporte a su hacer y que les permitan documentar para posteriormente compartir con otros docentes.

Dar continuidad y seguimiento a las acciones propuestas hasta evaluarlas será otro elemento a ser tomado en cuenta, porque permitirá a los docentes vislumbrar las posibilidades que tiene el proponer acciones que se materialicen y se concreten en la mejora del servicio. 


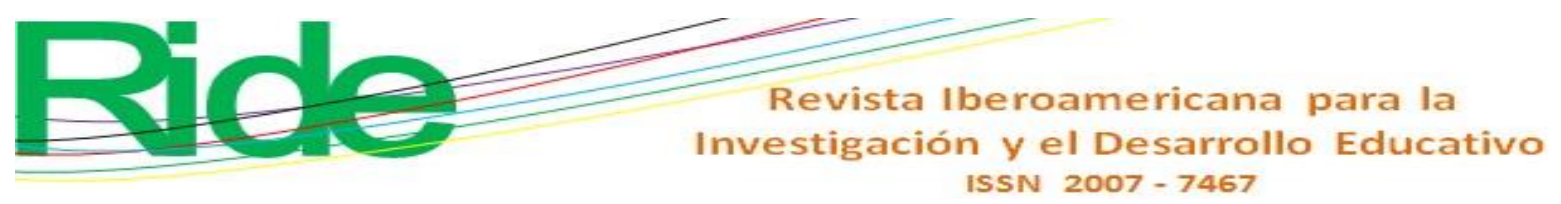

Esto los empodera, hace visible la posibilidad de aportar, de recuperar el conocimiento que les da la experiencia.

Lo anterior, además, genera arraigo en la institución, en la comunidad escolar y apertura la posibilidad de compartir la experiencia con otros, lo cual, a su vez, promueve la investigación desde el hacer docente a partir de propuestas como la investigación-acción y la intervención educativa, en la cual el profesional de la educación reconoce su potencial y la fuerza que tiene el análisis de su trabajo.

Asimismo, resulta indispensable reconocer la importancia de gestión para conseguir los fines propuestos. Esto implica que los responsables de las instituciones estén comprometidos con el desarrollo de sus docentes, y conseguir no solamente dar cuenta de resultados, sino también generar condiciones que les permitan empoderarse, poner en el centro inquietudes y necesidades.

Los planes de carrera es otro de los elementos a los que vale la pena voltear la vista, con la finalidad de que los docentes reconozcan y tomen control de lo que espera sea su vida profesional en la institución a la que están adscritos y de ser así en otros espacios. También se vislumbra la necesidad de que los docentes tomen consciencia de forma progresiva de la necesidad de la habilitación o capacitación que requieren en función de lo que ellos evalúan como prioritario para la mejora continua.

\section{Discusión}

En diversos espacios educativos se ha asociado de forma directa la habilitación, capacitación y superación profesional con el desarrollo profesional. En esta propuesta se vincula no como un sinónimo, pero sí como un elemento que sea previsto. En este sentido, se recuperan los principios de la educación de adultos desde diferentes propuestas teóricas. A decir de Knowles, Holton III y Swanson (1998), la educación de adultos deberá garantizar los siguientes principios: 


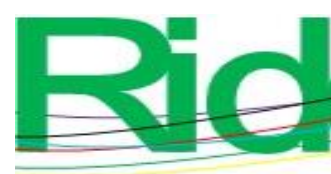

1. Autonomía.

2. Interactividad.

3. Adhesión o misión.

4. Aplicación inmediata.

5. Respeto a la necesidad de saber.

6. Respeto al concepto de sí mismo del discente.

Siguiendo con este planteamiento, se enfatiza que si bien es cierto que el aprendizaje es un proceso que se asegura a partir de la autodeterminación de quien desea aprender, en el aprendizaje de adultos, en específico cuando se hace alusión a la formación continua de los docentes, en su mayoría es una actividad extra que se vincula con las que de forma regular tienen a su cargo.

Se reconocen tres propuestas vinculadas con el aprendizaje de adultos para el desarrollo profesional. Porque, aunque es cierto que no es lo único, sí resulta un aspecto del desarrollo personal que se articula con indicadores para valorar a las instituciones educativas. En la tabla 1 se reconocen las propuestas andragógicas, humanistas y por competencias. 
Tabla 1. Teorías que sustentan el aprendizaje en adultos

\begin{tabular}{|c|c|c|}
\hline Teoría & Aportación & Autores \\
\hline Andragógica & La experiencia es un & Linderman (1925) \\
recurso de aprendizaje. Se & Knowles (1968) \\
relaciona con los resultados & Hiemstra (1993) \\
que consiga de forma & \\
& inmediata. Su & \\
& disponibilidad, por su parte, & \\
& se relaciona con los & \\
& beneficios potenciales. & \\
\hline Humanista & El aprendizaje se facilita. Es & \\
& significativo cuando las & \\
& personas reconocen que les & \\
& ayudarán a mantener la & \\
& estructura del yo. & \\
\hline Competencias & Se vislumbra como la forma & \\
& de desempeñar mejor & \\
& alguna labor o actividad. & \\
& &
\end{tabular}

Fuente: Elaboración propia con base en Cázares (2007)

Conocer estos elementos da la pauta para que las instituciones y los docentes tengan presente como un primer aspecto previo a la capacitación el hecho de detectar las necesidades. Debido a que esto permitirá al docente otorgar la importancia de los procesos de capacitación y habilitación. Y de esto modo también pugnará para que las propuestas tengan la eficacia buscada.

Hasta aquí los aspectos descritos tienen una relación directa y puntual con las instituciones educativas. Sin embargo, si se recupera el término sugerido para definir el desarrollo profesional, resulta necesario reconocer que para conseguir los resultados 
esperados es necesario promover el ámbito personal. Al respecto, es pertinente la idea de Nias (citado en Day, 2007):

Los maestros tienen corazón y cuerpo, igual que cabeza y manos, aunque la naturaleza rebelde de sus corazones está regida por sus cabezas, por la responsabilidad moral que tiene hacia los estudiantes y la integridad de su asignatura que está en el centro de su integridad profesional. No pueden enseñar bien si alguna parte de ellos se desentiende durante mucho tiempo (...). Si el equilibrio entre sentimiento, pensamiento y acción se trastorna en exceso o durante demasiado tiempo la enseñanza se distorsiona, las respuestas se reducen, incluso pueden dejar de ser capaces de enseñar. Los docentes están emocionalmente comprometidos con muchos aspectos de su ocupación (p. 30)

La labor docente, como se pone de manifiesto en la cita anterior, requiere de un equilibrio entre el sentimiento, el pensamiento y la acción. Sin duda lo anterior invita a reflexionar sobre qué tanto los docentes consiguen equilibrar los elementos mencionados. Desde las propuestas teóricas, las organizaciones que brindan servicios dependen de forma sustancial de los operadores, debido a que la experiencia de quien es el usuario se incrementa, disminuye o fracasa en función de la capacitación, potencialidades y metas de los integrantes de la organización.

La docencia implica, además, un reto. No solo se oferta un servicio, sino que los profesionales de la educación se convierten en un engranaje fundamental en el desarrollo de los infantes, adolescentes y adultos, en el caso de las instituciones formadoras. 
Tabla 2. Grado de adaptación del servicio

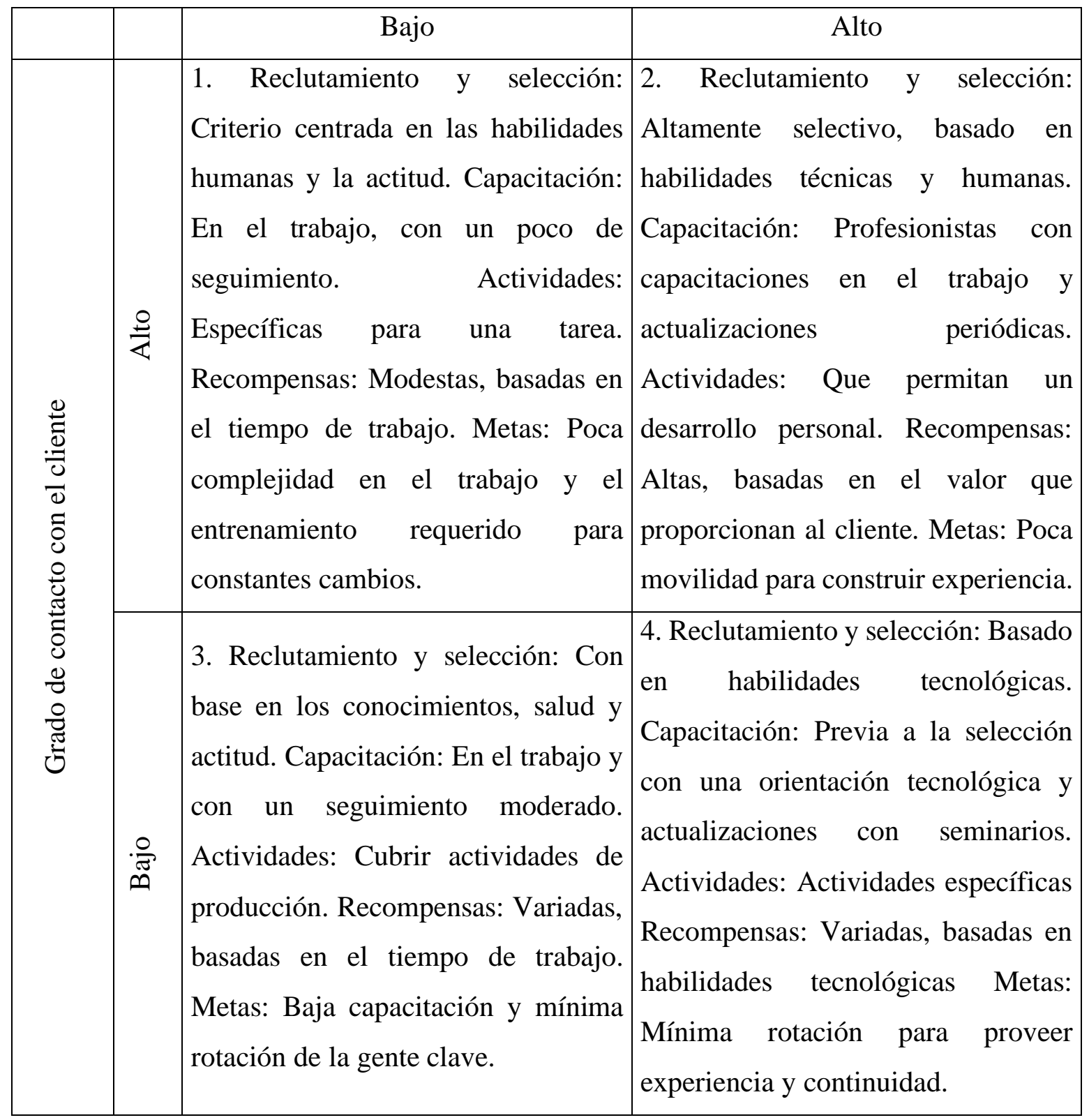

Fuente: Elaboración propia con base Heskett, Sasser y Hart (1990)

La tabla 2 explicita a partir de criterios básicos la necesidad de reconocer los elementos sustanciales a considerar en organizaciones de servicios. Sin perder de vista lo mencionado con antelación respecto a que las instituciones educativas van más allá, estos representarían los aspectos mínimos a considerar. 

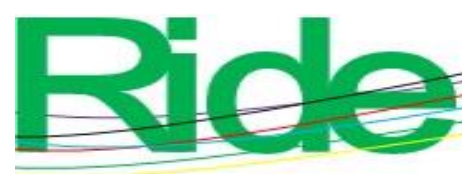

Revista Iberoamericana para la Investigación y el Desarrollo Educativo ISSN $2007-7467$

Un aspecto que recupera el segundo cuadrante, y que tiene que ver con el servicio profesional, es el de actividades que permitan el desarrollo personal. Y un concepto asociado a este es el de la satisfacción laboral. Aquí la satisfacción laboral se entiende de acuerdo con lo siguiente:

Un concepto multidimensional y multidisciplinar que supone el estado emocional, actitud, sensación o grado de bienestar que experimenta un individuo hacia su trabajo como consecuencia de la mayor o menor discrepancia existente entre sus expectativas pasadas y presentes acerca de las recompensas y el rol que le ofrece su empleo y la media en que ésta se cumplen realmente (Sánchez, Artacho, Fuentes y López, 2007, p. 869).

Si bien el término inició a ser considerado en los años 30, se reconocen antecedentes en propuestas como las de Taylor (1900; citado en Valverde, 2009), quien refería que el salario es una recompensa y que esta es una de las motivaciones que lleva a los trabajadores al cumplimiento de los objetivos. Otras propuestas como la de las relaciones humanas ponen de manifiesto los factores psicológicos y sociales, los cuales incluso tienen un mayor impacto que el factor salarial.

El concepto de satisfacción laboral recupera tres aspectos que permiten a las personas determinar si se sienten satisfechos o no, a saber, las expectativas, las recompensas y los roles que desempeñan en la organización. Vroom (1964; citado en Valverde, 2009) reconoció que la motivación puede venir de dos variables: la valencia y la expectativa. La primera tiene que ver con los resultados y la expectativa de alcanzar los resultados en función del esfuerzo realizado. En las propuestas de Vroom y de Porter Lawler existen elementos que se vinculan con lo que se espera, el esfuerzo que se hace y el desempeño, los cuales permiten alcanzar la satisfacción.

Cuando en la propuesta para definir el desarrollo profesional se hace alusión a la satisfacción de necesidades personales, profesionales y sociales, se reconoce la importancia de que el docente valore su labor social y cómo su trabajo trasciende con las generaciones que se forman. Esto asociado con procesos de evaluación auténtica que le permitan reconocer los logros y que, a partir de la retroalimentación, se consiga mejorar.

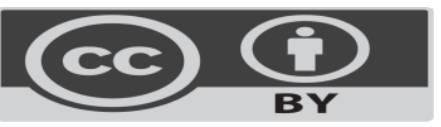


En este sentido, la importancia de aquilatar los aciertos de los maestros, de valorar sus esfuerzos en pro de la mejora educativa, así como de hacer visible su trabajo, es primordial. En un fragmento del juramento de los docentes que egresan de las escuelas normales públicas del Estado de México se afirma que "ser docente es encontrarse el hombre bajo la responsabilidad del mismo hombre".

Tarea que no es menor y por lo cual se requiere de un proceso de empoderamiento del docente; un proceso en el que se sienta capaz de tomar decisiones que le permitan desenvolverse mejor como profesional de la educación, que reconozca sus saberes, que colabore con otros para perfeccionar su tarea de manera conjunta, en el que un alumno que es capaz de crecer en lo personal como en lo académico sea un logro que subsane las horas de trabajo extra y la falta incluso de reconocimiento social que en países como el nuestro está latente.

Pensar en este sentido lleva a comprender que el desarrollo profesional de los docentes va más allá de perfeccionar la enseñanza, que tiene que ver con el desarrollo conjunto del que enseña con el que aprende, que reconoce la necesidad del ser humano de acompañar a otro, que trabaja en sus sentimientos y emociones para generar ambientes de aprendizaje propicios, en los que en ocasiones hay que olvidar el dolor propio para convertir el salón en un mundo mágico de aprendizaje.

Desde esta propuesta compleja, la docencia trae consigo fortalecer al docente para que sea capaz de dar todo aquello que se pretende desarrollar en los alumnos tanto en lo personal como en lo profesional, lo que a su vez implica que se responsabilice de su ciudadanía y que promueva en los otros las bases para que lo logren. Se reconoce que un ciudadano comprometido con su entorno es aquel que cuida de sí, de los otros y de los espacios; que sabe relacionarse en sus diferentes facetas, que contribuye para el desarrollo de los otros.

En este sentido, resulta necesario que las instituciones promuevan ambientes de aprendizaje que lo permitan, espacios en los cuales los maestros sean reconocidos, donde puedan opinar y desarrollar al máximo sus potencialidades; espacios para el trabajo en conjunto y de momentos para contrastar ideas que redunden en la tarea educativa. 
A partir de estas propuestas resultaría necesario encontrar opciones que, si bien no se conviertan en pasos o recetas, si den pauta para orientar a las instituciones y a los educadores respecto a cómo potenciar las acciones para promover de manera continua su desarrollo profesional.

Como hasta ahora se ha establecido, es necesario tener en cuenta que, desde esta visión, el eje nodal es el reconocimiento del docente tanto de manera personal como de forma institucional. Para ello se recupera la idea de formación que invita a cada uno de los actores educativos a que reconozca lo que quiere llegar a ser y, en consecuencia, realice acciones para conseguirlo. Esta postura implica un proceso de análisis y reflexión de parte de cada uno de los docentes. Pero, debido a que no siempre es una práctica común, las instituciones pueden generar alternativas en las que de manera conjunta se consiga vislumbrar estas perspectivas.

Para ello, se vislumbra la posibilidad de considerar la construcción de un plan de desarrollo profesional personalizado, en el que el docente reconozca cuál es el perfil profesional que desea alcanzar y determine los tiempos y formas para lograrlo. Este plan se complementa con el reconocimiento de las habilidades, fortalezas, preferencias personales y profesionales para establecer así un plan de carrera.

A partir de la autoevaluación que cada uno de los docentes realice con la finalidad de generar sinergias con la institución, será preponderante que el área responsable promueva las condiciones para alcanzar el desarrollo profesional de los docentes, revise el reconocimiento que el profesional de la educación efectúa y lo articule con las posibilidades y requerimientos institucionales para generar un plan común en el que se valore que entre mayor sea la satisfacción, personal y profesional de los docentes con respecto a la tarea que desempeñan, se obtendrá un mayor efecto significativo en el desarrollo institucional.

Una vez que se estableció el plan de carrera de cada uno de los profesionales de la educación, será conveniente delinear los medios para dar seguimiento y reflexionar de qué forma las acciones permiten los propósitos establecidos, a qué habría que darle continuidad y qué requiere transformarse, y pugnar con todo ello a que el docente consiga reconocer de qué forma se están cumpliendo sus expectativas.

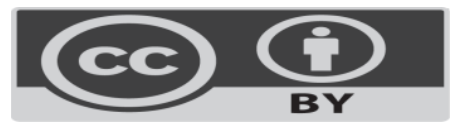



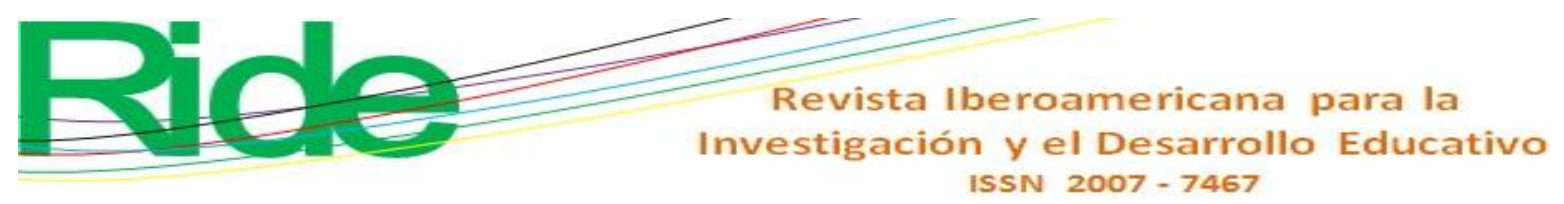

Conclusiones

El desarrollo profesional abre la posibilidad de potenciar el trabajo docente. Vale la pena pensar en cómo concebirlo. Si solamente se vislumbra como sinónimo de capacitación y actualización, estaría siendo visto como un proceso lineal, lo cual no es del todo cierto, debido a que con los mismos insumos no siempre se obtienen los mismos resultados, en este caso, ante las mismas condiciones para capacitación no siempre se obtienen los mismos resultados.

Vale la pena también reconocer que en los procesos de transformación y mejora es necesario no perder de vista que es el docente quien se sitúa en el centro de la transformación y mejora de su práctica, motivo por el cual los espacios para la reflexión y el análisis tendrán un papel determinante en el logro de los objetivos.

Además, debido a que la docencia no se desarrolla en aislado, resulta pertinente no perder de vista que en los espacios de trabajo entre pares se promueva el diálogo no solamente para acordar acciones o cumplir metas, que, pese a ser un aspecto importante y necesario, no se convierta en el centro y eje de las reuniones colegiadas; sino que a partir de los planes que se diseñen las problemáticas identificadas, las historias de éxito, se promuevan y generen momentos para fundamentar, analizar y reflexionar.

La propuesta de estos espacios se centra en consolidar comunidades de aprendizaje que permitan a quienes son parte de las instituciones construir conocimiento empírico, para lo cual se requiere promover su empoderamiento, y dar pauta con ello a que quienes son los responsables de promover los espacios educativos consigan generar alternativas tanto en lo personal como en lo profesional.

Como se hace notar, la propuesta que se presenta recupera la idea de que el desarrollo profesional docente es un proceso complejo, que vale la pena analizar, reconocer y potenciar, tratando de no encontrar soluciones únicas, sino más bien reconociendo los contextos, necesidades y posibilidades, pugnando por que los docentes sean cada vez más plenos y que, en consecuencia, su labor sea significativa para dar sentido a lo que hacen cotidianamente. 


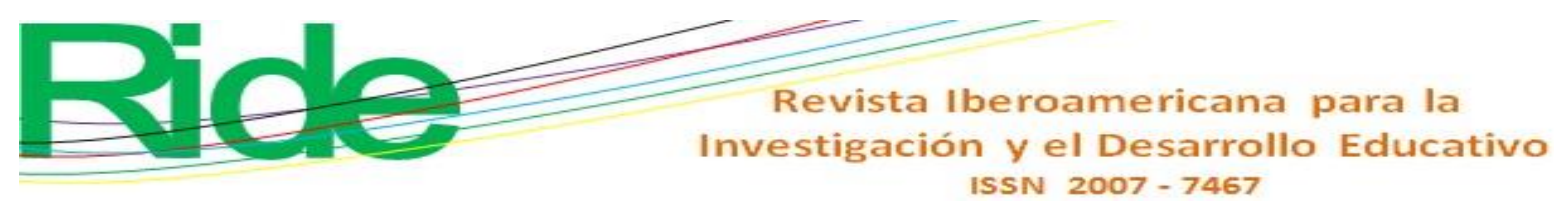

\section{Referencias}

Ackoff, R. (1999). Re-Creating the Corporation: A Design of Organization for the 21st Century. Nueva York, United States: Oxford University Press.

Cázares, Y. (2007). Aprendizaje Autodirigido en Adultos. Un modelo para su desarrollo. México: Trillas.

Day, C. (2007). Pasión por enseñar. La identidad personal y profesional del docente y sus valores (2. ${ }^{\mathrm{a}}$ ed.) (trad. P. Manzano) Madrid, España: Narcea.

Guber, R. (2011). La etnografía, método, campo y reflexividad. Buenos Aires, Argentina: Siglo Veintiuno Editores.

Heskett, J., Sasser, W. and Hart C. (1990) Service Breakthroughs. New York, United States: Simon and Schuster

Kaufman, R. (2008). Guía práctica para la planeación de organizaciones (trad. A. R. Casnati). Ciudad de México, México: Trillas.

Knowles, M. S., Holton III, E. F. and Swanson, R. A. (1998). The future of Andragogy. In Knowles, M. S., Holton III, E. F. and Swanson, R. A., The Adult Learner (6 ${ }^{\text {th }}$ ed.). San Diego, United States: Elsevier.

López, M. E. (2013). El desarrollo profesional de los formadores de docentes: Visto como un proceso complejo. Manuscrito no publicado. Estado de México, México: Instituto Universitario Internacional de Toluca.

Morin, E. (1990). Introducción al pensamiento complejo (trad. M. Pakman). Barcelona, España: Gedisa.

Prigogine, I. (1999). Las leyes del caos (trad. J. Vivánco). Barcelona, España: Crítica.

Rossett, A. (2002). Training Needs Assessment ( $9^{\text {th }}$ ed.). United States: Education Technology Publications, Inc.

Sánchez, S. M., Artacho, C., Fuentes, F. J. y López, T. J. (2007). Análisis de los Determinantes Estructurales de la Satisfacción Laboral. Aplicación en el Sector Educativo. Estudios de Economía Aplicada, 25, 867-900.

Valverde, M. (2009). Comportamiento humano en la organización. Barcelona, España: Universitat Oberta de Catalunya.

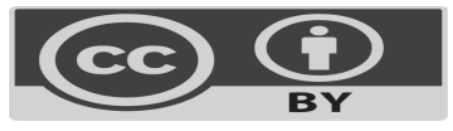

